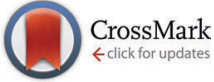

Cite this: Phys. Chem. Chem. Phys., 2015, 17, 9687

Received 11th January 2015, Accepted 10th February 2015

DOI: $10.1039 / c 5 c p 00101 c$

www.rsc.org/pccp

\title{
Excited-state intramolecular proton transfer to carbon atoms: nonadiabatic surface-hopping dynamics simulations $\uparrow$
}

\author{
Shu-Hua Xia, ${ }^{a}$ Bin-Bin Xie, ${ }^{a}$ Qiu Fang, ${ }^{a}$ Ganglong Cui*a and Walter Thiel*b
}

\begin{abstract}
Excited-state intramolecular proton transfer (ESIPT) between two highly electronegative atoms, for example, oxygen and nitrogen, has been intensely studied experimentally and computationally, whereas there has been much less theoretical work on ESIPT to other atoms such as carbon. We have employed CASSCF, MS-CASPT2, RI-ADC(2), OM2/MRCl, DFT, and TDDFT methods to study the mechanistic photochemistry of 2-phenylphenol, for which such an ESIPT has been observed experimentally. According to static electronic structure calculations, irradiation of 2-phenylphenol populates the bright $S_{1}$ state, which has a rather flat potential in the Franck-Condon region (with a shallow enol minimum at the CASSCF level) and may undergo an essentially barrierless ESIPT to the more stable $\mathrm{S}_{1}$ keto species. There are two $\mathrm{S}_{1} / \mathrm{S}_{0}$ conical intersections that mediate relaxation to the ground state, one in the enol region and one in the keto region, with the latter one substantially lower in energy. After $\mathrm{S}_{1} \rightarrow \mathrm{S}_{0}$ internal conversion, the transient keto species can return back to the $S_{0}$ enol structure via reverse ground-state hydrogen transfer in a facile tautomerization. This mechanistic scenario is verified by $\mathrm{OM} 2 / \mathrm{MRCl}$-based fewest-switches surface-hopping simulations that provide detailed dynamic information. In these trajectories, ESIPT is complete within $118 \mathrm{fs}$; the corresponding $\mathrm{S}_{1}$ excited-state lifetime is computed to be $373 \mathrm{fs}$ in vacuum. Most of the trajectories decay to the ground state via the $S_{1} / S_{0}$ conical intersection in the keto region (67\%), and the remaining ones via the enol region (33\%). The combination of static electronic structure computations and nonadiabatic dynamics simulations is expected to be generally useful for understanding the mechanistic photophysics and photochemistry of molecules with intramolecular hydrogen bonds.
\end{abstract}

\section{Introduction}

Excited-state intramolecular or intermolecular proton transfers are elementary processes occurring in many molecular and biochemical systems ${ }^{1-5}$ and electronic devices, ${ }^{6-9}$ for example, in natural and artificial photosynthesis, ${ }^{10,11}$ water-splitting photocatalysis, ${ }^{12}$ green fluorescent proteins, ${ }^{13,14}$ and photoswitches. ${ }^{15}$ Understanding these excited-state proton transfer processes is important both from fundamental and technological points of view. To this end, numerous computational studies ranging from static electronic structure calculations to nonadiabatic dynamics simulations have been performed in the past few decades. ${ }^{16-30}$ Most of these previous studies

\footnotetext{
${ }^{a}$ Key Laboratory of Theoretical and Computational Photochemistry, Ministry of Education, College of Chemistry, Beijing Normal University, Beijing 100875, China. E-mail: ganglong.cui@bnu.edu.cn

${ }^{b}$ Max-Planck-Institut für Kohlenforschung, 45470 Mülheim an der Ruhr, Germany. E-mail: thiel@mpi-muelheim.mpg.de

$\dagger$ Electronic supplementary information (ESI) available: Active space in OM2/ MRCI computations and Cartesian coordinates of all structures. See DOI: $10.1039 / \mathrm{c} 5 \mathrm{cp} 00101 \mathrm{c}$
}

focused on excited-state proton transfer processes between two highly electronegative atoms, e.g. nitrogen, oxygen, and fluorine. ${ }^{17-20,22,26,27,31-37}$

What about excited-state intramolecular proton transfer (ESIPT) in molecules without strong hydrogen bonds, for example, in alcohols or phenols? Such ESIPT processes were first investigated in the $1980 \mathrm{~s},{ }^{38,39}$ with proton transfer to aromatic carbon atoms being first addressed at the beginning of this century. ${ }^{40}$ Since then, Wan and coworkers have systematically explored such excited-state proton transfers in many systems. ${ }^{41-48}$ They first studied photochemical deuterium incorporation at the ortho and para positions of 2-phenylphenol in various solvent mixtures $^{49}$ and found that the predominant exchange at the ortho position is independent of water and methanol contents, implying an intramolecular process. They also investigated the photochemistry of $o$-hydroxybiaryls, which features not only an efficient excited-state proton transfer to the ortho carbon atom of the naphthyl ring, but also a novel ring-closing reaction. ${ }^{50}$ Flegel et $a .^{51}$ studied the photoaddition of water and alcohols to the 9- and 10-positions of the anthracene moiety of 9-(2-hydroxyphenyl)anthracene in acetonitrile and methanol mixtures and proposed a 
mechanism involving water-mediated excited-state proton transfer from the phenolic $\mathrm{OH}$ group to the anthracene fragment. Basaric and $\mathrm{Wan}^{52}$ investigated the potential excited-state proton transfer in four derivatives of 9-(2-hydroxyphenyl)anthracene. Nayak and Wan ${ }^{53}$ explored photochemical deuterium incorporation in extended orthosubstituted biaryl systems and reported the longest solvent-assisted proton-relay chain. They proposed direct and water-assisted proton transfer mechanisms to explain photohydration at the ortho and distal positions, respectively. In these experimental studies, it was generally believed that excited-state intramolecular proton transfer to ortho positions is efficient in phenols.

The underlying photophysical and photochemical mechanisms in these systems have not yet been elucidated in detail, e.g., with regard to the relevant structures, proton transfer paths, excited-state potential energy surfaces, lifetimes, and decay channels. We are aware of only one recent theoretical study in this context, ${ }^{54}$ which employed the single-reference second-order coupled cluster (RI-CC2) method to explore direct and water-assisted excited-state proton transfer in 2-phenyl1-naphthol. Given this situation, we decided to perform high-level multi-reference electronic structure computations and trajectory-based surface-hopping dynamics simulations to study the mechanistic photochemistry of the prototypical 2-phenylphenol molecule, with emphasis on the ESIPT process to the ortho carbon atom and the deactivation channels leading back to the ground state.

\section{Computational details}

\section{$A b$ initio methods}

Ground-state $\left(\mathrm{S}_{0}\right)$ conformers were optimized at the B3LYP level. ${ }^{55-58}$ The resolution-of-the-identity second-order algebraic diagrammatic construction [RI-ADC(2)] method was employed to optimize excited-state minimum-energy reaction paths. ${ }^{59-63}$

The state-averaged complete active space self-consistent field (SA-CASSCF) method (equal state weights) was used to optimize minima $\left(\mathrm{S}_{0}\right.$ and $\left.\mathrm{S}_{1}\right)$ and minimum-energy conical intersections $\left(\mathrm{S}_{0} / \mathrm{S}_{1}\right)$. In all SA-CASSCF geometric optimizations, the active space comprised 10 electrons in 8 orbitals. To obtain more accurate potential energy profiles, single-point MS-CASPT2 calculations ${ }^{64,65}$ were performed at the CASSCF optimized geometries. In these MS-CASPT2 calculations, an imaginary shift of 0.2 a.u. was applied to avoid intruder-state issues, ${ }^{66}$ and Cholesky decomposition techniques with unbiased auxiliary basis sets were used to evaluate two-electron integrals. ${ }^{67}$

Vertical excitation energies were computed at the TD-CAMB3LYP ${ }^{68,69}$ and MS-CASPT2 levels. The 6-31G* basis set $^{70,71}$ was used throughout except for the RI-ADC(2) calculations which employed the def2-SVP basis set. ${ }^{72}$ The following codes were used: TDDFT, GAUSSIAN09; ${ }^{73}$ DFT and CASSCF optimizations, GAUSSIAN03 $;^{74}$ MS-CASPT2, MOLCAS7.6; ${ }^{75}$ and RI-ADC(2), TURBOMOLE6.5. ${ }^{76}$

\section{OM2/MRCI method}

All semiempirical calculations were performed using the OM2/ MRCI method as implemented in the MNDO99 code. $^{77-80}$
During geometry optimizations, all required energies, gradients, and nonadiabatic coupling elements were computed analytically. Conical intersections were optimized using the Lagrange-Newton approach. ${ }^{81,82}$

In OM2/MRCI calculations, the restricted open-shell $\mathrm{HF}$ formalism was applied in the self-consistent field (SCF) treatment (i.e., the orbitals were optimized for the leading configuration of the $S_{1}$ state with two singly occupied orbitals). The active space in the MRCI calculations included 12 electrons in 10 orbitals (see ESI $\dagger$ ). In terms of the SCF configuration, it comprised the five highest doubly occupied orbitals, two singly occupied orbitals, and the three lowest unoccupied orbitals. For the MRCI treatment, three configuration state functions were chosen as references, namely the SCF configuration and the two closed-shell configurations derived therefrom (i.e., all singlet configurations that can be generated from the HOMO and the LUMO of the closed-shell ground state). The MRCI wavefunction was built by allowing all single and double excitations from these three references.

The nonadiabatic dynamics was studied by performing 1 ps OM2/MRCI trajectory surface-hopping simulations. The initial atomic coordinates and velocities were randomly selected from 5 ps trajectories of ground-state molecular dynamics. The number of excited-state dynamics runs was then chosen according to the computed $\mathrm{S}_{0}-\mathrm{S}_{1}$ transition probability. A total of 193 surface-hopping trajectories were run, with all relevant energies, gradients, and nonadiabatic coupling vectors being computed on-the-fly as needed. For points with an $S_{1}-S_{0}$ energy gap of less than $10 \mathrm{kcal} \mathrm{mol}^{-1}$, the fewest-switches criterion was applied to decide whether to hop. The time step was chosen to be 0.1 fs for nuclear motion and 0.0005 fs for electronic propagation. The unitary propagator evaluated at a mid-point was used to propagate the electronic motion. The translational and rotational motions were removed in each step. The empirical decoherence correction (0.1 a.u.) proposed by Granucci et al. was employed. ${ }^{83}$ The final evaluations were done for the 148 trajectories that finished successfully and satisfied our energy continuity criterion (no changes greater than $30 \mathrm{kcal} \mathrm{mol}^{-1}$ between any two consecutive MD steps). Further technical details are given in our previous publications. ${ }^{26-29,84-86}$

\section{Results}

\section{Ground-state properties and vertical excitation energies}

Apart from the most stable ground-state structure of 2-phenylphenol (S0-ENOL), there is also a minimum for the keto tautomer (S0-KETO), see Fig. 1 and Table 1. For each of the two minima, OM2/MRCI and CASSCF yield similar geometries. S0-ENOL is more stable than S0-KETO by 34.7 (33.7) $\mathrm{kcal} \mathrm{mol}^{-1}$ at the OM2/MRCI (MS-CASPT2) level.

The computed vertical excitation energies for the first excited singlet state $\left(S_{1}\right)$ are collected in Table 2. The OM2/ MRCI value of $4.92 \mathrm{eV}$ agrees very well with the results from MS-CASPT2 (4.99 eV) and TD-CAM-B3LYP (4.93 eV). The calculations are consistent with the experimental value of $4.66 \mathrm{eV}$ 

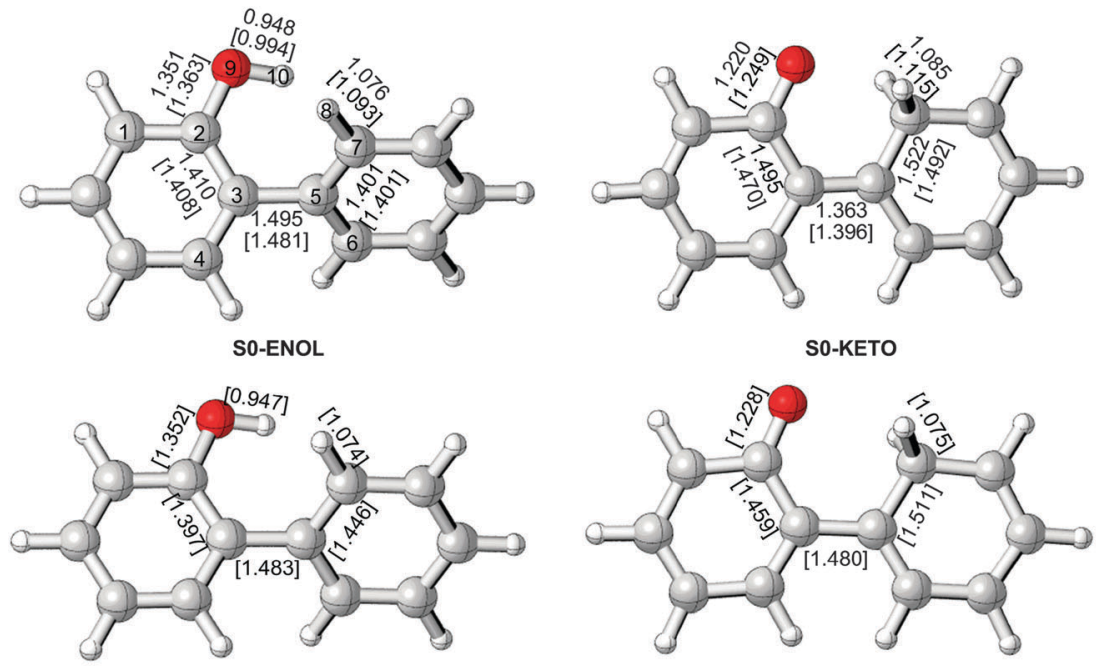

S1-ENOL
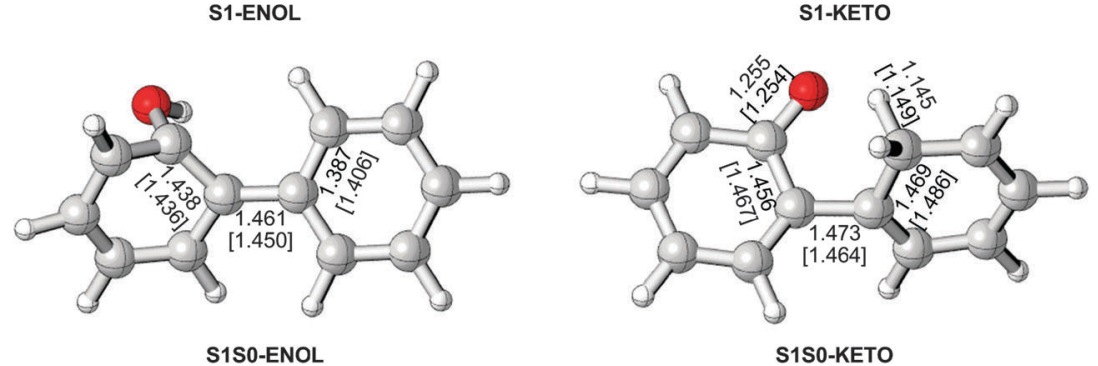

Fig. 1 Stationary points and minimum-energy conical intersections, with selected optimized bond lengths ( $\AA$ ) obtained from OM2/MRCI and CASSCF (in square brackets).

Table 1 Key dihedral angles (degree) of 2-phenylphenol structures from $\mathrm{OM} 2 / \mathrm{MRCl}$ and CASSCF optimizations and relative energies $\Delta E\left(\mathrm{kcal} \mathrm{mol}^{-1}\right)$ from $\mathrm{OM} 2 / \mathrm{MRCl}$ and single-point MS-CASPT2 calculations

\begin{tabular}{lcrrrr}
\hline Structure & C4C3C2O9 & C2C3C5C6 & C2C3C5C7 & C3C1C2O9 & \multicolumn{1}{l}{$\Delta E$} \\
\hline OM2/MRCI & & & & & \\
S0-ENOL & 179.6 & -127.7 & 52.8 & 179.7 & 0.0 \\
S0-KETO & 180.0 & 180.0 & 0.0 & 180.0 & 34.7 \\
S1S0-ENOL & -83.1 & 154.0 & -27.8 & 140.6 & 92.1 \\
S1S0-KETO & 166.8 & -107.9 & 58.2 & -171.7 & 73.7 \\
& & & & & \\
CASSCF(10,8)/6-31G* & & & & \\
S0-ENOL & 179.0 & -118.1 & 62.6 & 179.7 & 0.0 \\
S0-KETO & -180.0 & 179.8 & -0.2 & -180.0 & 33.7 \\
S1-ENOL & 178.2 & -132.3 & 51.2 & 179.4 & 103.0 \\
S1-KETO & 180.0 & 180.0 & 0.0 & 180.0 & 74.4 \\
S1S0-ENOL & -96.8 & 145.9 & -35.4 & 154.8 & $98.8 / 100.7$ \\
S1S0-KETO & 172.4 & -113.7 & 52.8 & -174.7 & $69.8 / 73.9$
\end{tabular}

Table 2 Computed vertical excitation energies to the first excited singlet state of 2-phenylphenol and the experimental band maximum from laser flash photolysis in solution ${ }^{49}$

\begin{tabular}{lllll}
\hline & OM2/MRCI & MS-CASPT2 & TD-CAM-B3LYP & Exp. \\
\hline $\mathrm{kcal} \mathrm{mol}^{-1}$ & 113.5 & 115.0 & 113.8 & 107.5 \\
$\mathrm{eV}$ & 4.92 & 4.99 & 4.93 & 4.66
\end{tabular}

obtained from laser flash photolysis of 2-phenylphenol in solutions. ${ }^{49}$ A slightly lower experimental value of $4.28 \mathrm{eV}$ has been reported for 2-phenyl-1-naphthol featuring more

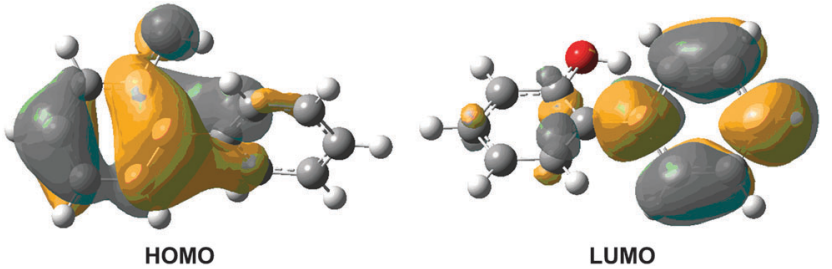

Fig. 2 CAM-B3LYP/6-31G* computed HOMO and LUMO of SO-ENOL responsible for the $S_{0} \rightarrow S_{1}$ vertical excitation.

extensive conjugation. ${ }^{54}$ The $\mathrm{S}_{1}$ state at the Franck-Condon point is spectroscopically bright; its oscillator strength is computed to be 0.155 at the TD-CAM-B3LYP level. Molecular orbital analysis shows that the $\mathrm{S}_{0}-\mathrm{S}_{1}$ electronic transition mainly originates from the HOMO-LUMO single excitation (Fig. 2). The HOMO is mainly localized in the phenolic part, whereas the LUMO is localized in the phenyl group. Hence, the $S_{1}$ state is of charge-transfer character, which sets the stage for the subsequent excited-state proton transfer. In fact, this kind of electronic structure change has been found in many similar intramolecularly hydrogen-bonded systems. ${ }^{18,20,22,26,27,30}$

\section{Excited-state minima}

At the CASSCF level, there is a shallow $\mathrm{S}_{1}$ minimum in the Franck-Condon region of 2-phenylphenol (S1-ENOL), which is computed to lie $103.0 \mathrm{kcal} \mathrm{mol}^{-1}$ above the S0-ENOL minimum in single-point MS-CASPT2 calculations. At the OM2/MRCI 

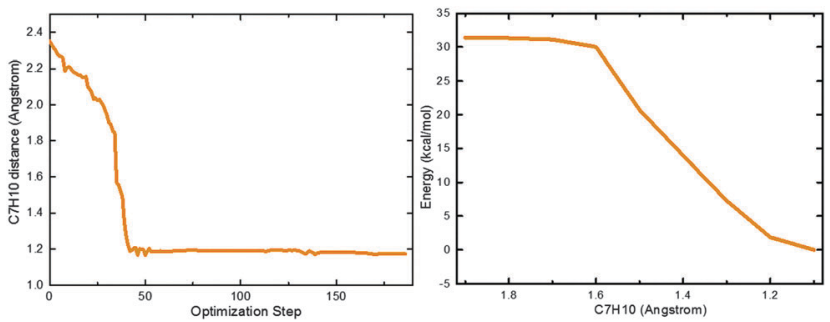

Fig. 3 (left) $\mathrm{OM} 2 / \mathrm{MRCl}$ optimization path starting from the enol minimum, which leads directly to a keto species after about 50 steps; (right) $\mathrm{RI}-\mathrm{ADC}(2) /$ def2-SVP computed minimum-energy reaction path with respect to the $\mathrm{C} 7-\mathrm{H} 10$ proton transfer reaction coordinate. See text for discussion.

level, no such $S_{1}$ minimum could be located since all minimizations starting from the S0-ENOL equilibrium geometry led directly to the $S_{1}$ keto species (S1-KETO), see the left panel of Fig. 3. Likewise, the minimum-energy path for proton transfer computed at the RI-ADC(2)/def2-SVP level indicates an essentially barrierless excited-state enol-keto tautomerization, see the right panel of Fig. 3. This is also verified by OM2/MRCI nonadiabatic dynamics simulations (vide infra).

CASSCF optimization yields another $S_{1}$ minimum in the keto region (S1-KETO, see Fig. 1). At this geometry, there is no significant charge transfer in the $S_{1}$ state; the $S_{0} \rightarrow S_{1}$ transition involves mostly the central $\mathrm{C} 3=\mathrm{C} 5$ double-bond region, causing an elongation of this bond from 1.396 to $1.480 \AA\left(\mathrm{S}_{0}\right.$ versus $\mathrm{S}_{1}$ keto minimum, CASSCF values). Other geometric parameters change only slightly (Fig. 1). According to single-point MS-CASPT2 calculations, S1-KETO lies $74.4 \mathrm{kcal}$ $\mathrm{mol}^{-1}$ above S0-ENOL and $28.6 \mathrm{kcal} \mathrm{mol}^{-1}$ below S1-ENOL (Table 1). Thus, the excited-state proton transfer that yields the keto species is highly exothermic; in other words, 2-phenylphenol is a strong photoacid in $\mathrm{S}_{1}$. As already mentioned, this proton transfer is computed to be essentially barrierless and is thus expected to be ultrafast. In terms of excited-state topology, our present results are consistent with recent RI-CC2 computations on a similar system, 2-phenyl-1naphthol. ${ }^{54}$

\section{Conical intersections}

At the OM2/MRCI level, we were able to locate two $S_{1} / S_{0}$ minimum-energy conical intersections (S1S0-ENOL and S1S0KETO). Selected bond lengths and dihedral angles are given in Fig. 1 and in Table 1, respectively. In S1S0-ENOL, the H10 atom is still attached to the $\mathrm{O} 9$ atom (phenol species) but the $\mathrm{OH}$ group is extruded out of the ring plane, with a $\mathrm{C} 4 \mathrm{C} 3 \mathrm{C} 2 \mathrm{O} 9$ dihedral angle of $-83^{\circ}$ (OM2/MRCI). This strong out-of-plane deformation significantly increases the $\mathrm{S}_{0}$ energy, thus closing the $\mathrm{S}_{0}-\mathrm{S}_{1}$ energy gap and reaching an $\mathrm{S}_{1} / \mathrm{S}_{0}$ conical intersection. In S1S0-KETO, the $\mathrm{H} 10$ atom is already bonded to the $\mathrm{C} 7$ atom (keto species); the two rings are not coplanar with a $\mathrm{C} 2 \mathrm{C} 3 \mathrm{C} 5 \mathrm{C} 7$ dihedral angle of $58^{\circ}$. Energetically, S1S0-ENOL [S1S0-KETO] is computed to lie 92.1 [73.7] $\mathrm{kcal} \mathrm{mol}^{-1}$ above the S0-ENOL ground state, and $21.4 \mathrm{kcal} \mathrm{mol}^{-1}\left[39.8 \mathrm{kcal} \mathrm{mol}^{-1}\right.$ ] below the $\mathrm{S}_{1}$ energy at the Franck-Condon point (113.5 $\left.\mathrm{kcal} \mathrm{mol}^{-1}\right)$; hence, these two conical intersections are energetically accessible. Taking into consideration that S1S0-KETO is more stable than S1S0-ENOL by $18.4 \mathrm{kcal} \mathrm{mol}^{-1}$, the former is expected to play a more vital role in excited-state deactivation.

It is worth stressing that OM2/MRCI and the $a b$ initio methods give similar structures and energies for the two $\mathrm{S}_{1} / \mathrm{S}_{0}$ conical intersections (Table 1 ). Taking S1S0-ENOL as an example, the dihedral angles $\mathrm{C} 2 \mathrm{C} 3 \mathrm{C} 5 \mathrm{C} 7, \mathrm{C} 4 \mathrm{C} 3 \mathrm{C} 2 \mathrm{O} 9$, and C3C1C2O 9 are computed to be $-28^{\circ},-83^{\circ}$, and $141^{\circ}$ at the OM2/MRCI level, compared with $-35^{\circ},-97^{\circ}$, and $155^{\circ}$ at the CASSCF level, respectively, (see Fig. 1). The relative energies from OM2/MRCI and single-point MS-CASPT2 calculations are also reasonably close to each other: the values of S1S0-ENOL [S1S0-KETO] are 92.1 [73.7] $\mathrm{kcal} \mathrm{mol}^{-1}$ for OM2/MRCI, and 98.8/100.7 [69.8/73.9] $\mathrm{kcal} \mathrm{mol}^{-1}$ for MS-CASPT2. In the latter case, the quoted $S_{0}$ and $S_{1}$ state energies differ slightly because they come from single-point MS-CASPT2 calculations at CASSCF-optimized geometries.

\section{Excited-state decay paths}

The preceding static electronic structure computations suggest the following scenario for the photoinduced processes in 2-phenylphenol. Upon irradiation, the spectroscopically bright $\mathrm{S}_{1}$ state is populated in the Franck-Condon region, from which the $S_{1} / S_{0}$ conical intersection with an intact phenol moiety is energetically accessible (with relaxation to the ground state via S1S0-ENOL). A competitive process involves an essentially barrierless excited-state proton transfer yielding an $S_{1}$ keto minimum, which can decay to the ground state via the $S_{1} / S_{0}$ conical intersection in the keto region (S1S0-KETO); back in the $\mathrm{S}_{0}$ state, the keto species S0-KETO can return to the more stable tautomer S0-ENOL via reverse ground-state hydrogen transfer.

To verify this mechanism and to explore the timescales of the underlying photophysical and photochemical events, we have performed trajectory-based fewest-switches surface-hopping dynamics simulations starting in the $S_{1}$ state of 2-phenylphenol.

\section{Hopping-point distribution}

The $\mathrm{S}_{1}-\mathrm{S}_{0}$ hopping-point distribution extracted from all surfacehopping trajectories reflects the topology of the conical intersection seam. ${ }^{84}$ The two types of $\mathrm{S}_{1} / \mathrm{S}_{0}$ conical intersections in 2-phenylphenol, S1S0-ENOL and S1S0-KETO, clearly govern our nonadiabatic dynamics simulations. Fig. 4 depicts the distributions of the $\mathrm{C} 7 \mathrm{H} 10$ distance and the $\mathrm{C} 4 \mathrm{C} 3 \mathrm{C} 2 \mathrm{O} 9$ dihedral angle
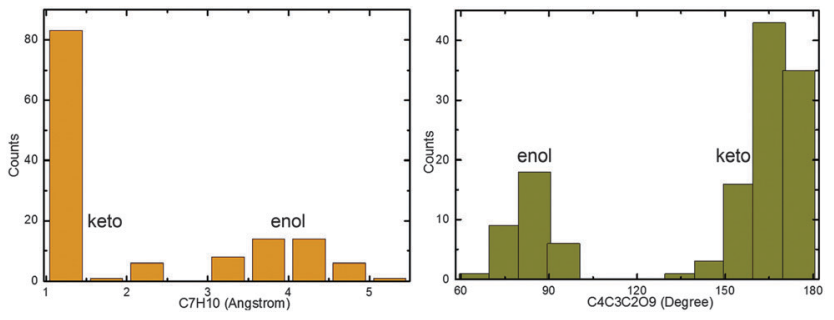

Fig. 4 Distribution of the $\mathrm{C} 7 \mathrm{H} 10$ distance and the $\mathrm{C} 4 \mathrm{C} 3 \mathrm{C} 2 \mathrm{C} 9$ dihedral angle at all $\mathrm{S}_{1}-\mathrm{S}_{0}$ hopping points. See text for detailed discussion. 
at all $\mathrm{S}_{1}-\mathrm{S}_{0}$ hopping points. Obviously, there are two main hopping regions, which cluster around two minimum-energy $\mathrm{S}_{1} / \mathrm{S}_{0}$ conical intersections S1S0-ENOL and S1S0-KETO. A closer examination of the $\mathrm{C} 7 \mathrm{H} 10$ distance distribution at all hopping points in Fig. 4 shows that most of the trajectories (67\%) hop to the $\mathrm{S}_{0}$ state via the keto conical intersection seam. This preference arises from two factors: first, the $S_{1}$ proton transfer is essentially barrierless so that the $S_{1}$ keto species is generated easily, and second, S1S0-KETO is thermodynamically favored over S1S0-ENOL because its potential energy is lower by $18.4 \mathrm{kcal} \mathrm{mol}^{-1}$ (OM2/MRCI). Hence, it is not surprising that most trajectories decay to the $\mathrm{S}_{0}$ state via S1S0-KETO in our dynamics simulations.

\section{$S_{1}$ lifetime}

In our simulations, 118 of $148(80 \%)$ trajectories have reached the $S_{0}$ state at the end of the 1 ps nonadiabatic dynamics runs. As shown in the left panel of Fig. 5, most of the $S_{1} \rightarrow S_{0}$ hops happen between 100 and $400 \mathrm{fs}$ (only 4 hops after $400 \mathrm{fs}$ ). Again, this ultrafast decay is consistent with the excited-state topological features, i.e. an almost barrierless proton transfer and two efficient deactivation channels (vide supra).

The $S_{1}$ excited-state deactivation can be viewed as a firstorder elementary reaction. The $S_{1}$ state population is thus ruled by the following rate equation:

$$
p(t)=\exp \left(-k\left(t-t_{0}\right)\right)+p_{0}
$$

where $k$ is the corresponding rate constant; $p_{0}$ is the $S_{1}$ population at the end of the run ( 0.2 in this work); and $t_{0}$ is the initial delay time (57 fs). After fitting the time-dependent state population in Fig. 4 to eqn (1), we obtain an $S_{1}$ excitedstate lifetime of $373 \mathrm{fs}$. One should note that the $\mathrm{S}_{1}$ excited-state lifetime may be expected to increase in the condensed phase, in particular in a rigid environment.

\section{Product distribution}

Fig. 6 shows the product distribution at the end of the 1 ps nonadiabatic simulations. Overall, there are four kinds of products, namely $\mathrm{S}_{1}$ enol (13\%) and keto (5\%), and $\mathrm{S}_{0}$ enol $(47 \%)$ and keto $(35 \%)$; the enol : keto ratio is estimated to be $3: 2$. The top panel illustrates the distribution of the resulting phenol conformers. Most of the trajectories ending up in the phenol region have the $\mathrm{H} 10$ atom bonded to the $\mathrm{O} 9$ atom. However, there are also some $S_{0}$ phenol products that have the
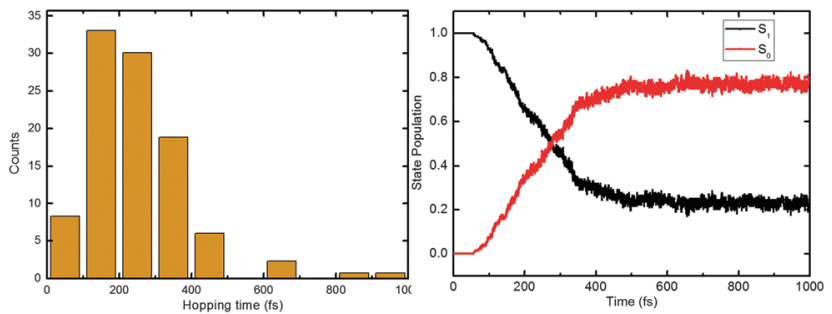

Fig. 5 Distribution of the $S_{1}-S_{0}$ hopping times (left) and time-dependent $\mathrm{S}_{1}$ and $\mathrm{S}_{0}$ state populations (right). See text for detailed discussion.
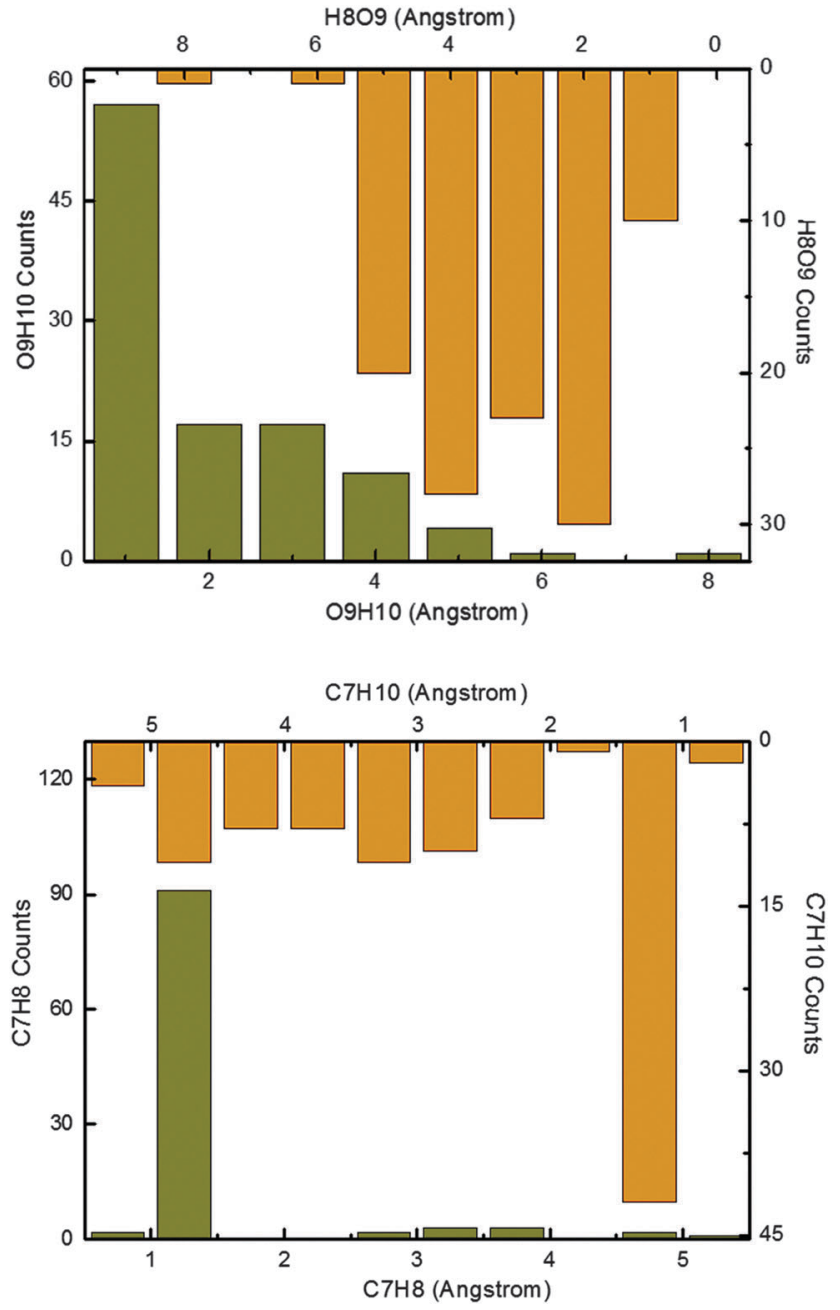

Fig. 6 Distribution of the $\mathrm{C} 7 \mathrm{H} 10, \mathrm{C} 7 \mathrm{H} 8, \mathrm{H} 8 \mathrm{O} 9$, and $\mathrm{O} 9 \mathrm{H} 10$ bond lengths at the end of 1 ps simulations. See text for detailed discussion.

$\mathrm{H} 8$ atom bonded to the $\mathrm{O} 9$ atom, not the $\mathrm{H} 10$ atom (see $\mathrm{H} 8 \mathrm{O} 9$ distribution). In these trajectories, the excited-state proton transfer and the reverse ground-state hydrogen transfer involve two different hydrogen atoms (H10 and H8, respectively). The bottom panel depicts the distribution of the keto conformers after the 1 ps simulations. There are ca. 90 trajectories with the $\mathrm{H} 8$ atom bonded to the $\mathrm{C} 7$ atom, and $c a$. 40 trajectories with the $\mathrm{H} 10$ atom bonded to the $\mathrm{C} 7$ atom.

\section{Typical trajectories}

In our nonadiabatic dynamics simulations, we see three different photocycles that start from S1-ENOL and end up at S0-ENOL: (I) the $S_{1}$ state decays directly to the ground state, without excitedstate intramolecular proton transfer (ESIPT); (II) the $S_{1}$ state first evolves towards the $S_{1}$ keto species via an ultrafast barrierless ESIPT and then decays to the ground state in the keto region followed by a reverse ground-state hydrogen transfer (GSHT) involving the same migrating hydrogen atom; (III) the photocycle is the same as in case (II) except that different hydrogen atoms are involved in ESIPT and GSHT. In the following, we present for each 

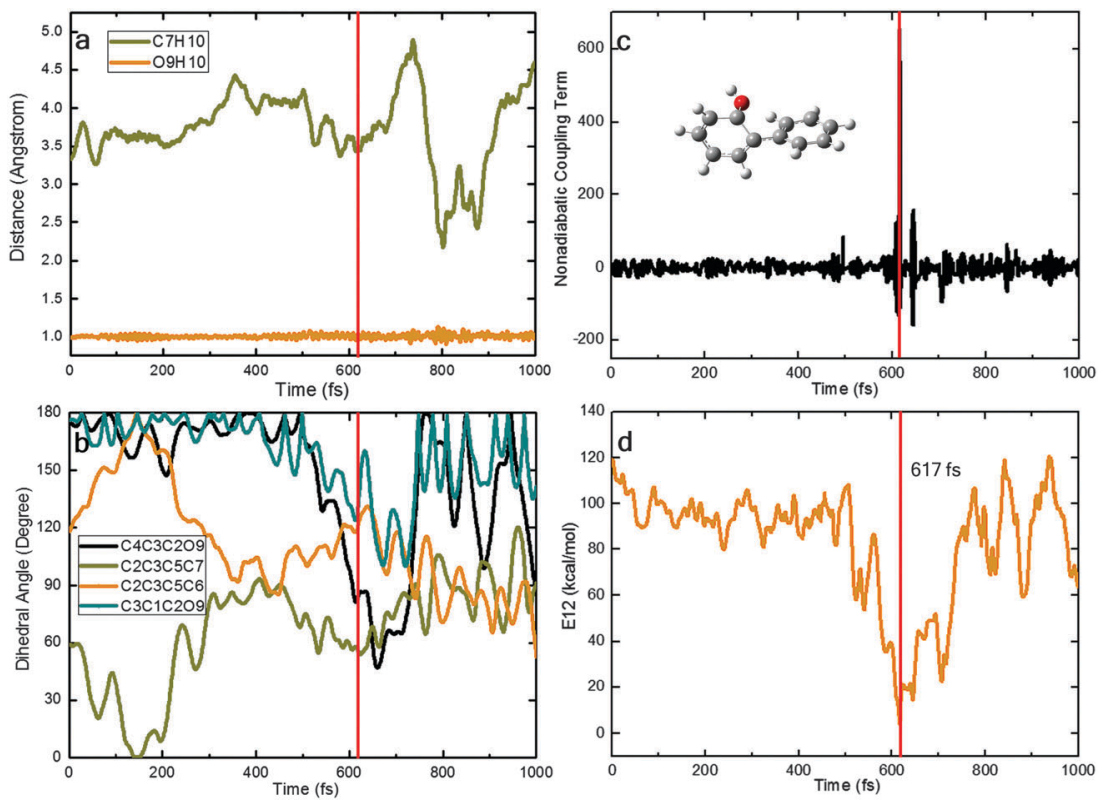

Fig. 7 Time-dependent physical variables obtained from a typical OM2/MRCI trajectory of type (I): (a) two key bond lengths; (b) four key dihedral angles; (c) nonadiabatic coupling term; and (d) $\mathrm{S}_{1}-\mathrm{S}_{0}$ energy gap.

photocycle pattern a representative trajectory to illustrate the main photophysical and photochemical events.

Fig. 7 shows a typical trajectory for case (I) with direct decay via the S1S0-ENOL conical intersection. Within the first $400 \mathrm{fs}$, the system starts to rotate around its central C3C5 bond (strong changes in the $\mathrm{C} 2 \mathrm{C} 3 \mathrm{C} 5 \mathrm{C} 6$ and $\mathrm{C} 2 \mathrm{C} 3 \mathrm{C} 5 \mathrm{C} 7$ dihedral angles; only small fluctuations in the $44 \mathrm{C} 3 \mathrm{C} 2 \mathrm{O} 9$ and C3C1C2O9 dihedral angles). During this process, the nonadiabatic coupling remains small and the $S_{1}-S_{0}$ energy gap remains large, so there is no nonadiabatic transition. After about $400 \mathrm{fs}$, the $\mathrm{C} 4 \mathrm{C} 3 \mathrm{C} 2 \mathrm{O} 9$ dihedral angle starts to decrease from $180^{\circ}$ to $40^{\circ}$ at $c a .600 \mathrm{fs}$. The $\mathrm{S}_{1}$ and $\mathrm{S}_{0}$ states now become energetically close to each other (within $4 \mathrm{kcal} \mathrm{mol}^{-1}$ ) and there is a large nonadiabatic coupling; thus, a nonadiabatic $\mathrm{S}_{1}-\mathrm{S}_{0}$ hop takes place, with relaxation of $\mathrm{S}_{1}$ to the $\mathrm{S}_{0}$ state. Thereafter, the $\mathrm{C} 4 \mathrm{C} 3 \mathrm{C} 2 \mathrm{O} 9$ and $\mathrm{C} 3 \mathrm{C} 1 \mathrm{C} 2 \mathrm{O} 9$ dihedral angles move back towards their original values (from twisted to a more planar arrangement). There is no ESIPT in this
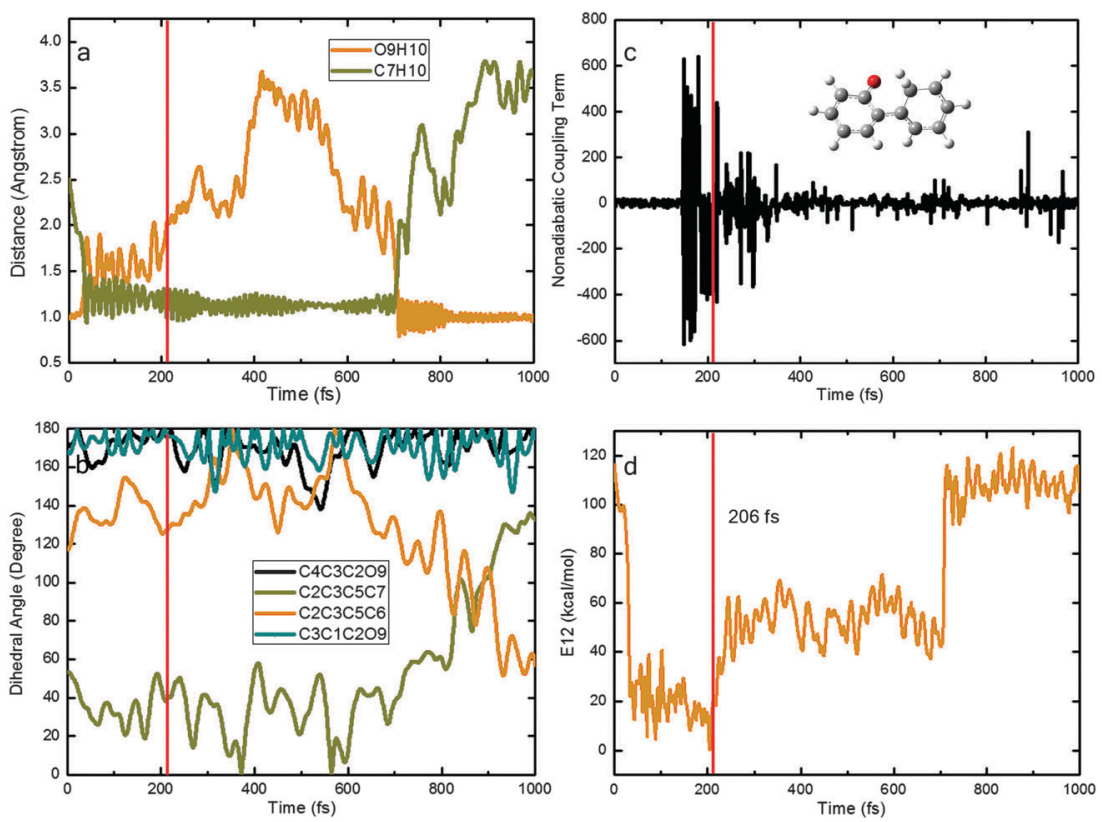

Fig. 8 Time-dependent physical variables obtained from a typical OM2/MRCI trajectory of type (II): (a) two key bond lengths; (b) four key dihedral angles; (c) nonadiabatic coupling term; and (d) $\mathrm{S}_{1}-\mathrm{S}_{0}$ energy gap. 
trajectory. We emphasize that this photocycle pattern occurs only rarely in our trajectories.

Fig. 8 depicts a typical trajectory for case (II) with deactivation to the $\mathrm{S}_{0}$ state via the S1S0-KETO conical intersection. In the initial stage of this trajectory, the $\mathrm{O} 9 \mathrm{H} 10$ and $\mathrm{C} 7 \mathrm{H} 10$ distances quickly increase and decrease, respectively. At ca. $50 \mathrm{fs}$, the ESIPT is complete and the $\mathrm{S}_{1}$ keto species $\mathrm{S} 1-\mathrm{KETO}$ is formed, which remains in the $\mathrm{S}_{1}$ state for another 150 fs (while retaining a rather short O9H10 distance indicative of excited-state hydrogen bonding interactions). Thereafter, it decays to the $S_{0}$ state at a point where the $S_{1}-S_{0}$ nonadiabatic coupling becomes very large (panel c) and the $S_{1}-S_{0}$ gap is very small (panel d). Interestingly, the generated $\mathrm{S}_{0}$ keto species does not return back to the enol region immediately; instead, it roams the keto region for additional 500 fs. Then, a reverse groundstate hydrogen transfer takes place, regenerating the $S_{0}$ enol conformer and completing the photocycle. The rotation around the $\mathrm{C} 3-\mathrm{C} 5$ bond starts after $\mathrm{ca}$. $700 \mathrm{fs}$ (see the C2C3C5C6 and $\mathrm{C} 2 \mathrm{C} 3 \mathrm{C} 5 \mathrm{C} 7$ dihedral angles in panel b) while the $\mathrm{C} 4 \mathrm{C} 3 \mathrm{C} 2 \mathrm{O} 9$ and C3C1C2O9 dihedral angles do not vary much.

Fig. 9 presents a typical trajectory for case (III). Here, the O9H10 and $\mathrm{C} 7 \mathrm{H} 10$ distances fluctuate around their equilibrium positions in the first $100 \mathrm{fs}$; then, they start to increase and decrease quickly. At about 110 fs, the $S_{1}$ keto species S1KETO is formed, which stays in the $\mathrm{S}_{1}$ state for $c a .50 \mathrm{fs}$ and then decays to the $S_{0}$ state at $165 \mathrm{fs}$, when the keto $S_{1} / S_{0}$ conical intersection is encountered. The generated keto species roams the keto region in the $S_{0}$ state for a longer time (640 fs). After ca. $800 \mathrm{fs}$, the most stable $\mathrm{S}_{0}$ phenol conformer is regenerated via a reverse ground-state hydrogen transfer. The rotation around the $\mathrm{C} 3-\mathrm{C} 5$ bond starts after ca. 900 fs (see the C2C3C5C6 and C2C3C5C7 dihedral angles in panel b). Interestingly, the $\mathrm{H} 10$ atom bonded to the $\mathrm{O} 9$ atom is transferred to the $\mathrm{C} 7$ atom in the ESIPT process, while the $\mathrm{H} 8$ atom originally bonded to the $\mathrm{C} 7$ atom is transferred to the $\mathrm{O} 9$ atom in the final GSHT step.

\section{Discussion}

Our results are consistent with the experiments available for 2-phenylphenol. Lukeman and $\mathrm{Wan}^{49}$ argued that singlet reactivity is major for 2-phenylphenol, which is consistent with our computations. The $\mathrm{S}_{1}$ excited-state proton transfer is nearly barrierless and ultrafast, so it is impossible for the system to efficiently populate triplet states in the Franck-Condon region. In addition, the $S_{1} \rightarrow T_{1}$ intersystem crossing in the keto region is not expected to be competitive with the efficient internal conversion from the $S_{1}$ keto species to the $S_{0}$ state. However, this intersystem crossing could become more probable in a rigid environment because the internal conversion involves a large conformational change that could be impeded by steric interactions with the environment. Furthermore, there is experimental evidence that 2-phenylphenol is a strong photoacid in the $S_{1}$ state. This point is supported by the MS-CASPT2 results (see Fig. 10), which confirm that the $S_{1}$ excited-state proton transfer is highly exothermic - S1-KETO lies $28.6 \mathrm{kcal} \mathrm{mol}^{-1}$ below S1-ENOL and $40.6 \mathrm{kcal} \mathrm{mol}^{-1}$ below the initially populated $\mathrm{S}_{1}$ Franck-Condon point.

We emphasize in this context that our present computations are carried out in vacuum and thus only consider the intrinsic photochemistry of 2-phenylphenol, for example, direct ESIPT processes to the ortho-position, without accounting for solventassisted intermolecular proton transfer to remote sites such as para-positions.
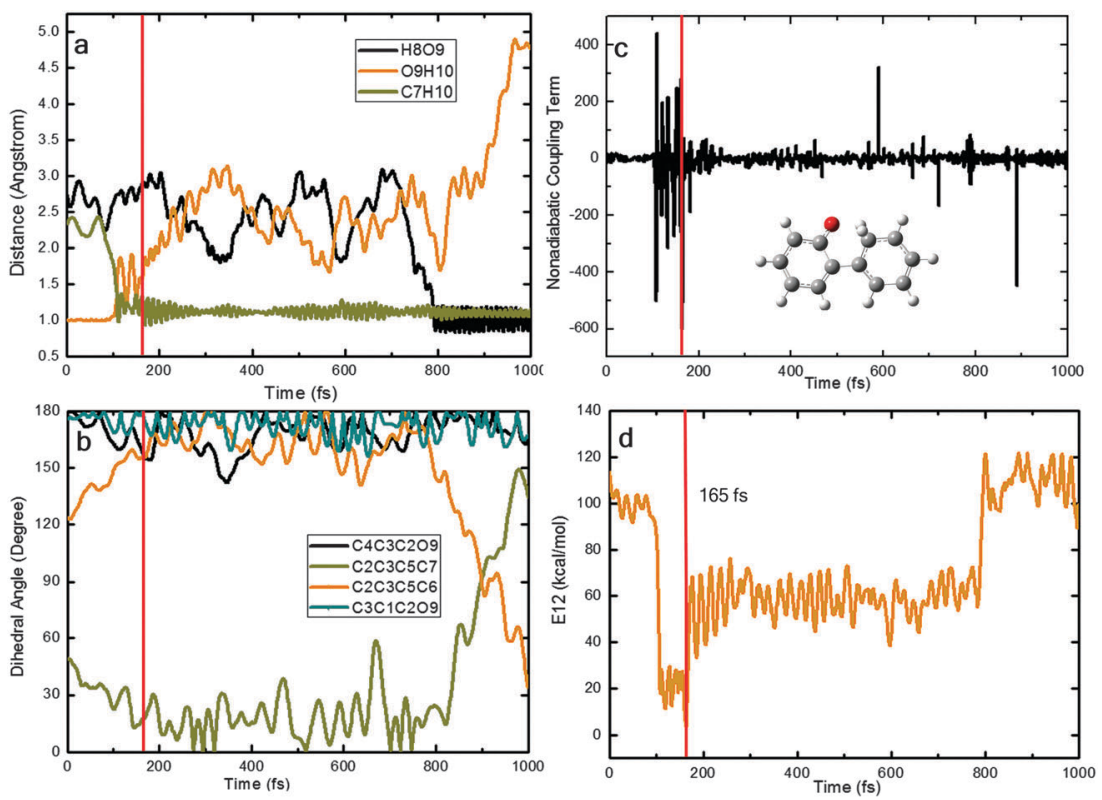

Fig. 9 Time-dependent physical variables obtained from a typical OM2/MRCl trajectory of type (III): (a) three key bond lengths; (b) four key dihedral angles; (c) nonadiabatic coupling term; and (d) $\mathrm{S}_{1}-\mathrm{S}_{0}$ energy gap. 

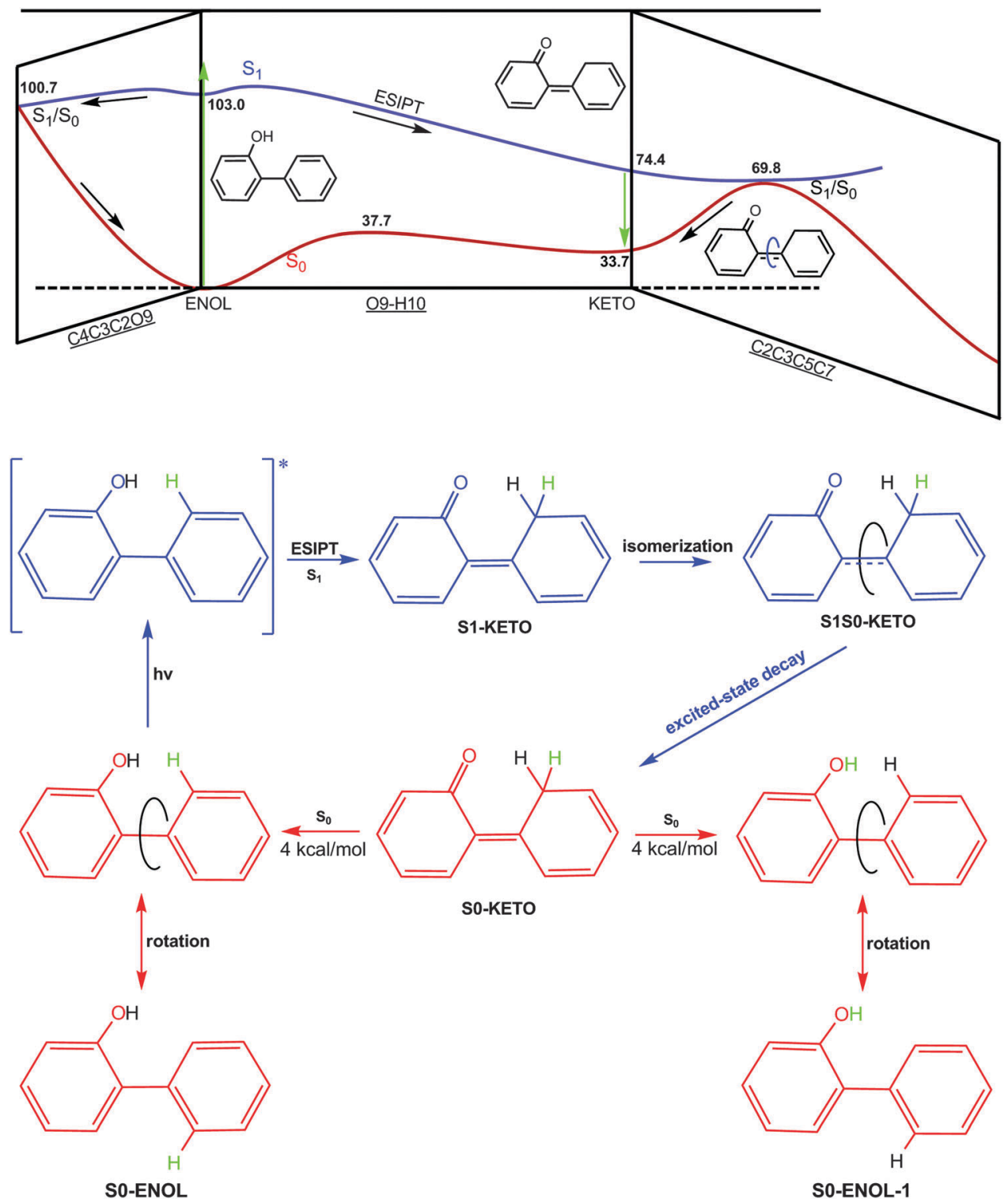

Fig. $10 \mathrm{~S}_{1}$ deactivation pathways identified in the present work. Potential energy profiles and structures related to the $\mathrm{S}_{0}$ and $\mathrm{S}_{1}$ states are shown in red and blue, respectively. Also relative energies from single-point MS-CASPT2 computations (in kcal mol ${ }^{-1}$ ) are given. See text for discussion.

Previous electronic structure computations on a similar system ${ }^{54}$ showed that there exists an efficient $S_{1} / S_{0}$ conical intersection near the keto region, but without optimizing its structure. In this work we precisely located this kind of minimum-energy conical intersection in 2-phenylphenol, both at the OM2/MRCI and CASSCF levels (S1S0-KETO), and we explored its dynamical role in the $\mathrm{S}_{1}$ photodynamics of 2-phenylphenol using full-dimensional surface-hopping dynamics simulations. We find that $67 \%$ trajectories decay to the $\mathrm{S}_{0}$ state via this conical intersection in the keto region. In addition, we optimized the $S_{1} / S_{0}$ conical intersection in the Franck-Condon region (S1S0-ENOL), which also plays an important role in the $S_{1}$ deactivation $(33 \%)$. Thus, both conical intersections need to be considered in order to correctly understand the mechanistic photochemistry of 2-phenylphenol and its variants.
How is the $\mathrm{S}_{0}$ isomer S0-ENOL-1 (see the bottom of Fig. 10) generated in the photodynamics of 2-phenylphenol? Experimentally, Lukeman and $\mathrm{Wan}^{49}$ assumed that this species comes from SO-KETO via a concerted reverse hydrogen transfer and 1,5hydrogen shift. Our present dynamics simulations do not support this scenario - we do not see any 1,5-hydrogen shift in any of the trajectories. Instead, S0-ENOL-1 is generated by a simple singlebond rotation, after the hydrogen atom originally bonded to the phenyl ring has been transferred to the oxygen atom (Fig. 10).

The $\mathrm{S}_{0}$ keto species has not yet been detected spectroscopically when using nanosecond laser flash photolysis. ${ }^{49,54}$ B3LYP calculations indicate that the tautomerization of S0-KETO to the most stable phenol conformer S0-ENOL has to overcome a small barrier of $4.0 \mathrm{kcal} \mathrm{mol}^{-1}$. It might thus be possible to observe this predicted transient species using ultrafast timeresolved transient spectroscopy. 


\section{Summary}

With the use of electronic structure computations and trajectory-based surface-hopping dynamics simulations, we have for the first time explored the mechanistic photochemistry of 2-phenylphenol. We have simulated the $S_{1}$ excited-state proton transfer and deactivation as well as the reverse hydrogen transfer in the $S_{0}$ state. Mechanistically, some trajectories directly evolve from the Franck-Condon region toward an enol-type $S_{1} / S_{0}$ conical intersection, followed by an $S_{1} \rightarrow S_{0}$ internal conversion to the ground-state minimum. Most of the trajectories proceed from the Franck-Condon region to the $\mathrm{S}_{1}$ keto species via an essentially barrierless ESIPT; the transient $\mathrm{S}_{1}$ keto species is then de-excited to the ground state via a second $\mathrm{S}_{1} / \mathrm{S}_{0}$ conical intersection in the keto region, followed by a quick relaxation back to the most stable phenol minimum via a reverse GSHT process (barrier of $c a .4 \mathrm{kcal} \mathrm{mol}^{-1}$ at the B3LYP level). The nonadiabatic dynamics simulations predict an average lifetime of $118 \mathrm{fs}$ for the ESIPT process. ${ }^{40,49}$ In these simulations, $67 \%$ of the trajectories decay to the $S_{0}$ state via the keto $\mathrm{S}_{1} / \mathrm{S}_{0}$ conical intersection, and $33 \%$ decay via the $\mathrm{S}_{1} / \mathrm{S}_{0}$ conical intersection in the Franck-Condon region. According to the computed time-dependent state populations, the $\mathrm{S}_{1}$ excitedstate lifetime is estimated to be $373 \mathrm{fs}$ in vacuum. We hope that these computational results and mechanistic insights will stimulate further experimental work on 2-phenylphenol, especially by ultrafast time-resolved transient spectroscopy.

\section{Acknowledgements}

G.C. appreciates the financial support of "The Recruitment Program of Global Youth Experts" and "Youth Scholars Program of Beijing Normal University"; W.T. is grateful for support from an ERC Advanced Grant.

\section{References}

1 E. Kosower and D. Huppert, Annu. Rev. Phys. Chem., 1986, 37, 127-156.

2 R. Mathies, S. Lin, J. Ames and W. Pollard, Annu. Rev. Biophys. Biophys. Chem., 1991, 20, 491-518.

3 R. Cukier and D. Nocera, Annu. Rev. Phys. Chem., 1998, 49, 337-369.

4 L. Tolbert and K. Solntsev, Acc. Chem. Res., 2002, 35, 19-27.

5 D. Stoner-Ma, A. Jaye, K. Ronayne, J. Nappa, S. Meech and P. Tonge, J. Am. Chem. Soc., 2008, 130, 1227-1235.

6 K. Choi and A. Hamilton, Angew. Chem., Int. Ed., 2001, 40, 3912-3915.

7 S. Kim, J. Seo, H. Jung, J.-J. Kim and S. Park, Adv. Mater., 2005, 17, 2077-2082.

8 S. Park, O.-H. Kwon, S. Kim, S. Park, M.-G. Choi, M. Cha, S. Park and D.-J. Jang, J. Am. Chem. Soc., 2005, 127, 10070-10074.

9 J. Kwon and S. Park, Adv. Mater., 2011, 23, 3615-3642.

10 A. Bard and M. Fox, Acc. Chem. Res., 1995, 28, 141-145.
11 D. Gust, T. Moore and A. Moore, Acc. Chem. Res., 2001, 34, 40-48.

12 W. Youngblood, S.-H. Lee, Y. Kobayashi, E. HernandezPagan, P. Hoertz, T. Moore, A. Moore, D. Gust and T. Mallouk, J. Am. Chem. Soc., 2009, 131, 926-927.

13 R. Tsien, Annu. Rev. Biochem., 1998, 67, 509-544.

14 K.-Y. Chen, Y.-M. Cheng, C.-H. Lai, C.-C. Hsu, M.-L. Ho, G.-H. Lee and P.-T. Chou, J. Am. Chem. Soc., 2007, 129, 4534-4535.

15 S.-J. Lim, J. Seo and S. Park, J. Am. Chem. Soc., 2006, 128, 14542-14547.

16 W.-H. Fang, J. Am. Chem. Soc., 1998, 120, 7568-7576.

17 A. Sinicropi, R. Pogni, R. Basosi, M. Robb, G. Gramlich, W. Nau and M. Olivucci, Angew. Chem., Int. Ed., 2001, 40, 4185-4189.

18 A. Sobolewski, W. Domcke and C. Hättig, Proc. Natl. Acad. Sci. U. S. A., 2005, 102, 17903-17906.

19 J. Coe and T. Martínez, J. Am. Chem. Soc., 2005, 127, 4560-4561.

20 A. Sobolewski and W. Domcke, J. Phys. Chem. A, 2007, 111, 11725-11735.

21 A. Migani, L. Blancafort, M. Robb and A. DeBellis, J. Am. Chem. Soc., 2008, 130, 6932-6933.

22 M. Barbatti, A. Aquino, H. Lischka, C. Schriever, S. Lochbrunner and E. Riedle, Phys. Chem. Chem. Phys., 2009, 11, 1406-1415.

23 F. Plasser, M. Barbatti, A. Aquino and H. Lischka, J. Phys. Chem. A, 2009, 113, 8490-8499.

24 D. Shemesh, A. Sobolewski and W. Domcke, J. Am. Chem. Soc., 2009, 131, 1374-1375.

25 S. Olsen, K. Lamothe and T. Martínez, J. Am. Chem. Soc., 2010, 132, 1192-1193.

26 G. Cui, Z. Lan and W. Thiel, J. Am. Chem. Soc., 2012, 134, 1662-1672.

27 G. Cui and W. Thiel, Phys. Chem. Chem. Phys., 2012, 14, 12378-12384.

28 L. Spörkel, G. Cui, A. Koslowski and W. Thiel, J. Phys. Chem. A, 2013, 118, 152-157.

29 L. Spörkel, G. Cui and W. Thiel, J. Phys. Chem. A, 2013, 117, 4574-4583.

30 G. Cui, P.-J. Guan and W.-H. Fang, J. Phys. Chem. A, 2014, 118, 4732-4739.

31 L. Serrano-Andrés and M. Merchán, Chem. Phys. Lett., 2006, 418, 569-575.

32 A. Kyrychenko and J. Waluk, J. Phys. Chem. A, 2006, 110, 11958-11967.

33 H.-H. G. Tsai, H.-L. S. Sun and C.-J. Tan, J. Phys. Chem. A, 2010, 114, 4065-4079.

34 G.-J. Zhao and K.-L. Han, Acc. Chem. Res., 2012, 45, 404-413. 35 Y. Shigemitsu, T. Mutai, H. Houjou and K. Araki, J. Phys. Chem. A, 2012, 116, 12041-12048.

36 H. Fang and Y. Kim, J. Chem. Theory Comput., 2013, 9, 3557-3566.

37 Y. Houari, A. Charaf-Eddin, A. D. Laurent, J. Massue, R. Ziessel, G. Ulrich and D. Jacquemin, Phys. Chem. Chem. Phys., 2014, 16, 1319-1321. 
38 M. Isaks, K. Yates and P. Kalanderopoulos, J. Am. Chem. Soc., 1984, 106, 2728-2730.

39 P. Kalanderopoulos and K. Yates, J. Am. Chem. Soc., 1986, 108, 6290-6295.

40 M. Lukeman and P. Wan, Chem. Commun., 2001, 1004-1005.

41 P. Wan and G. Zhang, Res. Chem. Intermed., 1993, 19, 119-129.

42 N. Basarić and P. Wan, Photochem. Photobiol. Sci., 2006, 5, 656-664.

43 N. Aein and P. Wan, J. Photochem. Photobiol., A, 2009, 208, 42-49.

44 N. Basarić, A. Franco-Cea, M. Alesković, K. MlinarićMajerski and P. Wan, Photochem. Photobiol. Sci., 2010, 9, 779-790.

45 Y.-H. Wang and P. Wan, Photochem. Photobiol. Sci., 2011, 10, 1934-1944.

46 N. Basarić, N. Doslić, J. Ivković, Y. H. Wang, J. Veljković, K. Mlinarić-Majerski and P. Wan, J. Org. Chem., 2013, 78, 1811-1823.

47 Y.-H. Wang and P. Wan, Photochem. Photobiol. Sci., 2013, 12, 1571-1588.

48 D. Skalamera, K. Mlinarić-Majerski, I. Martin-Kleiner, M. Kralj, P. Wan and N. Basarić, J. Org. Chem., 2014, 79, 4390-4397.

49 M. Lukeman and P. Wan, J. Am. Chem. Soc., 2002, 124, 9458-9464.

50 M. Lukeman and P. Wan, J. Am. Chem. Soc., 2003, 125, 1164-1165.

51 M. Flegel, M. Lukeman, L. Huck and P. Wan, J. Am. Chem. Soc., 2004, 126, 7890-7897.

52 N. Basarić and P. Wan, J. Org. Chem., 2006, 71, 2677-2686.

53 M. Nayak and P. Wan, Photochem. Photobiol. Sci., 2008, 7, 1544-1554.

54 N. Basarić, N. Doslić, J. Ivković, Y. H. Wang, M. Malis and P. Wan, Chem. - Eur. J., 2012, 18, 10617-10623.

55 S. Vosko, L. Wilk and M. Nusair, Can. J. Phys., 1980, 58, 1200-1211.

56 A. Becke, Phys. Rev. A: At., Mol., Opt. Phys., 1988, 38, 3098-3100.

57 C. Lee, W. Yang and R. Parr, Phys. Rev. B: Condens. Matter Mater. Phys., 1988, 37, 785-789.

58 A. Becke, J. Chem. Phys., 1993, 98, 1372-1377.

59 J. Schirmer, Phys. Rev. A: At., Mol., Opt. Phys., 1982, 26, 2395-2416.

60 A. Trofimov and J. Schirmer, J. Phys. B: At., Mol. Opt. Phys., 1995, 28, 2299-2324.

61 A. Köhn and C. Hättig, J. Chem. Phys., 2003, 119, 5021-5036.

62 C. Hättig, Adv. Quantum Chem., 2005, 50, 37-60.

63 A. Dreuw and M. Wormit, Wiley Interdiscip. Rev.: Comput. Mol. Sci., 2015, 5, 82-95.

64 K. Andersson, P. Malmqvist, B. Roos, A. Sadlej and K. Wolinski, J. Phys. Chem., 1990, 94, 5483-5488.

65 K. Andersson, P. Malmqvist and B. Roos, J. Chem. Phys., 1992, 96, 1218-1226.

66 N. Försberg and P. Malmqvist, Chem. Phys. Lett., 1997, 274, 196-204.
67 F. Aquilante, R. Lindh and T. Pedersen, J. Chem. Phys., 2007, 127, 114107-114713.

68 M. A. L. Marques, C. A. Ullrich, F. Nogueira, A. Rubio, K. Burke and E. K. U. Gross, Time-dependent Density Functional Theory, Springer, 2006.

69 T. Yanai, D. Tew and N. Handy, Chem. Phys. Lett., 2004, 393, 51-57.

70 R. Ditchfield, W. Hehre and J. Pople, J. Chem. Phys., 1971, 54, 724-728.

71 P. Hariharan and J. Pople, Theor. Chem. Acc., 1973, 28, 213-222.

72 A. Schäfer, H. Horn and R. Ahlrichs, J. Chem. Phys., 1992, 97, 2571-2577.

73 M. J. Frisch, G. W. Trucks, H. B. Schlegel, G. E. Scuseria, M. A. Robb, J. R. Cheesem, G. Scalmani, V. Barone, B. Mennucci, G. A. Petersson, H. Nakatsuji, M. Caricato, X. Li, H. P. Hratchian, A. F. Izmaylov, J. Bloino, G. Zheng, J. L. Sonnenberg, M. Hada, M. Ehara, K. Toyota, R. Fukuda, J. Hasegawa, M. Ishida, T. Nakajima, Y. Honda, O. Kitao, H. Nakai, T. Vreven, J. A. Montgomery Jr., J. E. Peralta, F. Ogliaro, M. Bearpark, J. J. Heyd, E. Brothers, K. N. Kudin, V. N. Staroverov, R. Kobayashi, J. Normand, K. Raghavachari, A. Rendell, J. C. Burant, S. S. Iyengar, J. Tomasi, M. Cossi, N. Rega, J. M. Millam, M. Klene, J. E. Knox, J. B. Cross, V. Bakken, C. Adamo, J. Jaramillo, R. Gomperts, R. E. Stratmann, O. Yazyev, A. J. Austin, R. Cammi, C. Pomelli, J. W. Ochterski, R. L. Martin, K. Morokuma, V. G. Zakrzewski, G. A. Voth, P. Salvador, J. J. Dannenberg, S. Dapprich, A. D. Daniels, O. Farkas, J. B. Foresman, J. V. Ortiz, J. Cioslowski and D. J. Fox, Gaussian 09, Revision A.02, Gaussian, Inc., Wallingford CT, 2009.

74 M. J. Frisch, G. W. Trucks, H. B. Schlegel, G. E. Scuseria, M. A. Robb, J. R. Cheeseman, J. A. Montgomery Jr., T. Vreven, K. N. Kudin, J. C. Burant, J. M. Millam, S. S. Iyengar, J. Tomasi, V. Barone, B. Mennucci, M. Cossi, G. Scalmani, N. Rega, G. A. Petersson, H. Nakatsuji, M. Hada, M. Ehara, K. Toyota, R. Fukuda, J. Hasegawa, M. Ishida, T. Nakajima, Y. Honda, O. Kitao, H. Nakai, M. Klene, X. Li, J. E. Knox, H. P. Hratchian, J. B. Cross, V. Bakken, C. Adamo, J. Jaramillo, R. Gomperts, R. E. Stratmann, O. Yazyev, A. J. Austin, R. Cammi, C. Pomelli, J. W. Ochterski, P. Y. Ayala, K. Morokuma, G. A. Voth, P. Salvador, J. J. Dannenberg, V. G. Zakrzewski, S. Dapprich, A. D. Daniels, M. C. Strain, O. Farkas, D. K. Malick, A. D. Rabuck, K. Raghavachari, J. B. Foresman, J. V. Ortiz, Q. Cui, A. G. Baboul, S. Clifford, J. Cioslowski, B. B. Stefanov, G. Liu, A. Liashenko, P. Piskorz, I. Komaromi, R. L. Martin, D. J. Fox, T. Keith, M. A. Al-Laham, C. Y. Peng, A. Nanayakkara, M. Challacombe, P. M. W. Gill, B. Johnson, W. Chen, M. W. Wong, C. Gonzalez and J. A. Pople, Gaussian 03, Revision D.02, Gaussian, Inc., Wallingford, CT, 2004.

75 F. Aquilante, L. De Vico, N. Ferré, G. Ghigo, P. Malmqvist, P. Neogrády, T. Pedersen, M. Pitonák, M. Reiher, B. Roos, L. Serrano-Andrés, M. Urban, V. Veryazov and R. Lindh, J. Comput. Chem., 2010, 31, 224-247. 
76 TURBOMOLE V6.5 2013, a development of University of Karlsruhe andForschungszentrum Karlsruhe $\mathrm{GmbH}$, 19892007, TURBOMOLE GmbH, since 2007; available from http://www.turbomole.com.

77 W. Weber, PhD thesis, University of Zürich, 1996.

78 W. Weber and W. Thiel, Theor. Chem. Acc., 2000, 103, 495-506.

79 A. Koslowski, M. E. Beck and W. Thiel, J. Comput. Chem., 2003, 24, 714-726.

$80 \mathrm{~W}$. Thiel, MNDO99 program, version 6.1, Max-Planck-Institut für Kohlenforschung, Mülheim, Germany, 2007.
81 D. Yarkony, J. Chem. Phys., 2001, 114, 2601-2613.

82 T. W. Keal, A. Koslowski and W. Thiel, Theor. Chem. Acc., 2007, 118, 837-844.

83 G. Granucci, M. Persico and A. Zoccante, J. Chem. Phys., 2010, 133, 134111-134119.

84 G. Cui and W. Thiel, Angew. Chem., Int. Ed., 2013, 52, 433-436.

85 O. Weingart, Z. Lan, A. Koslowski and W. Thiel, J. Phys. Chem. Lett., 2011, 2, 1506-1509.

86 A. Kazaryan, Z. Lan, L. Schäfer, W. Thiel and M. Filatov, J. Chem. Theory Comput., 2011, 7, 2189-2199. 